\title{
TESTES PSICOLÓgICOS: LEVANTAMENTO DOS MOTIVOS PELOS QUAIS RECEBERAM PARECER DESFAVORÁVEL SEGUNDO A COMISSÃO CONSULTIVA
}

\author{
Fernanda Andrade de Freitas \\ Doutoranda em Educação pelo Grupo de Pesquisa em Psicologia e Educação Superior - PES da \\ Universidade Estadual de Campinas/UNICAMP e Mestre em Avaliação Psicológica. \\ Lucicleide Cantalice \\ Docente do curso de Psicologia da Universidade São Francisco - USF e Mestre em Avaliação \\ Psicológica.
}

\begin{abstract}
Resumo
Este estudo teve como objetivo identificar os motivos, segundo a resolução do Conselho Federal de Psicologia - CFP n. ${ }^{\circ}$ 002/2003, pelos quais os testes psicológicos receberam Parecer desfavorável. A relação está disponível no site do Conselho Federal de Psicologia. Cinquenta e nove (59) pareceres dos testes reprovados foram impressos e analisados por dois juízes por meio de categorias estabelecidas. De modo geral, os resultados revelaram que o ano de 2003 contou com mais testes submetidos à avaliação e a editora, com mais testes reprovados, foi a Centro Editor de Psicologia Aplicada - CEPA e $(45,7 \%)$ dos testes psicológicos tinha problemas quanto à fundamentação teórica e à definição de construto. Uma minoria $(5,1 \%)$ apresentava estudos de validade e de precisão (13,6\%), mas a maioria apresentou estudos normativos $(67,8 \%)$, ainda que não estivessem totalmente adequados. Os dados foram discutidos em termos dos impactos positivos que a regulamentação gerou na área.
\end{abstract}

Palavras-chave: avaliação psicológica, testes psicológicos, medidas, psicologia.

\section{PSYCHOLOGICAL TESTING: SURVEY OF THE MOTIVES BY WHICH THEY HAVE RECEIVED UNFAVORABLE REPORT ACCORDING TO THE CONSULTING COMMISSION}

\begin{abstract}
This survey had the objective of identify the motives, according to the resolution of the Federal Council of Psychology - FCP no. 002/2003, why the psychological tests had received unfavorable report. The relation is available in the Federal Council of Psychology site. Fifty-nine (59) Reports of the rejected tests were printed and analyzed by two judges trough categories established. Overall, the results revealed that in 2003 there were more tests submitted to assessment and the publishing house with more rejected tests was Center Editor of Applied Psychology - CEPA and $(45,7 \%)$ of the psychological tests had problems due to the theoretical justification and to the construct definition. A minority $(5,1 \%)$ had presented studies of validity and efficiency $(13,6 \%)$, but the majority had presented normative studies $(67,8 \%)$ even they were not totally adequate. The data were discussed in means of positive impacts that the regulation had produced in the area.
\end{abstract}

Keywords: psychological assessment, psychological testing, measurements, psychology. 


\title{
TESTES PSICOLÓGICOS: ESTUDIO DE LAS RAZONES SEGUNDO RECIBIÓ UN DICTAMEN DESFAVORABLE DE LA COMISIÓN CONSULTIVA
}

\begin{abstract}
Resumen
Este estudio tuve el objetivo de identificar los motivos, segundo la resolución del Consejo Federal de Sicología - CFS - no. 002/2003, pelos cuales los testes psicológicos recibieran Parecer desfavorable. La relación está disponible en el site del Consejo Federal de Sicología. Cincuenta e nueve (59) pareceres de los testes reprobados fueran impresos y analizados por dos jueces por categorías establecidas. De modo general, los resultados rebelaran que el ano de 2003 a contado con más testes sometidos á la avaluación e la editora con más testes reprobados fue lo Centro Editor de Psicología Aplicada - CEPA e $(45,7 \%)$ de los testes psicológicos tenia problemas cuanto la fundamentación teórica e la definición del constructor. Una minoría $(5,1 \%)$ presentaba estudios de validad y de precisión $(13,6 \%)$, mas la mayoría presentaba estudios normativos $(67,8 \%)$ aunque non estuviesen totalmente adecuados. Los dados fueran discutidos en termos de los impactos positivos que la reglamentación engendró en la área.
\end{abstract}

Palabras clave: evaluación psicológica, testes psicológicos, medidas, psicología.

\section{INTRODUÇÃO}

A importância da avaliação psicológica (AP) para o profissional da área de psicologia acentua-se à medida que se afirma que é privativa desse profissional conforme a lei n. ${ }^{0} 4.119$ de 27/08/62 (alínea "a", parágrafo $1^{\circ}$, artigo 13). Ela se refere à coleta e interpretação de informações psicológicas, resultantes de um conjunto de procedimentos confiáveis que permitam ao Psicólogo avaliar o comportamento (Resolução CFP n.o 012/2000). A AP é um elemento importante na intervenção profissional, pois possibilita trazer informações relevantes acerca do problema, orientar medidas e proceder à avaliação dos resultados. Desse modo, o profissional que faz uso da AP deve apresentar tanto competência técnica e conduta ética como conhecimento a fim de interpretar adequadamente os resultados de sua avaliação. No caso da realização de pesquisas na área, para que o problema de pesquisa seja respondido, o pesquisador deve demonstrar a validade de sua interpretação e os critérios adotados para a tomada de decisão e assim, respeitar com rigor as etapas de realização de uma avaliação, pois é de suma importância para seu êxito (Oakland, 2004).

A AP é composta por quatro etapas sendo que a primeira pressupõe o levantamento das necessidades, comportamentos e aspectos psicológicos; a segunda, a integração das descrições e dos resultados; a terceira, a inferência e, por último, a intervenção (Pasquali, 2003). Sua realização se dá por meio da cientificidade, ou seja, por instrumentos de medida (testes psicológicos) que, por 
sua vez, devem apresentar parâmetros psicométricos que evidenciem sua eficácia (Urbina, 2007; Pasquali, 2003).

Historicamente, a AP foi evoluindo e, portanto, as suas características foram se diferenciando, em especial, a partir do ano 2000, pois passou a ser discutida em função das dificuldades do uso, construção e estabelecimento de critérios científicos a respeito dos testes psicológicos, ferramentas importantes ao processo de AP (Pasquali \& Alchieri, 2001). Nesse mesmo período, o Conselho Federal de Psicologia (CFP), por meio de comissões que visam regulamentar e fiscalizar a prática profissional, e algumas organizações profissionais, como sociedades e institutos, publicaram resoluções e/ou manifestos que visavam refletir acerca da prática profissional, a comercialização e a construção dos testes psicológicos. As reflexões se justificam na tentativa de minimizar o uso inadequado dos instrumentos, produzindo preconceitos e discriminação social; de avaliar os instrumentos comercializados desatualizados e sem embasamento científico; de revisar a produção de laudos inadequados e o uso exclusivamente técnico de instrumentos sem uma atitude crítico-reflexiva fundamentada teórica e cientificamente (Noronha, Freitas \& Ottati, 2002).

Alguns exemplos dessa mobilização são: 1. o manual para Avaliação Psicológica de candidatos à Carteira Nacional de Habilitação e condutores de veículos automotores (Resolução CFP n.0 012/00); 2. a definição do que é um teste psicológico como método de avaliação privativo do psicólogo e a regulamentação da sua elaboração, comercialização e uso (Resolução CFP n.o 025/01); 3. a regulamentação da avaliação Psicológica em Concurso Público e processos seletivos da mesma natureza (Resolução CFP n.0 01/2002); 4 . o trabalho do psicólogo na Avaliação Psicológica de candidatos à Carteira Nacional de Habilitação e condutores de veículos automotores (Resolução CFP n.o 016/2002); 5. a definição e regulamentação do o uso, a elaboração e a comercialização de testes psicológicos que revoga a Resolução CFP n. 0 025/2001 (Resolução CFP no 02/2003); 6. o manual de Elaboração de Documentos Escritos produzidos pelo psicólogo, decorrentes de Avaliação Psicológica (Resolução CFP n. ${ }^{0}$ 007/2003); 7. o trabalho do psicólogo na Avaliação Psicológica para concessão de registro e/ou porte de arma de fogo (Resolução CFP n.o 018/2008); 8. as normas e procedimentos para a Avaliação Psicológica no contexto do Trânsito e a revogação da Resolução CFP n. ${ }^{0}$ 012/2000 (Resolução CFP n.0 007/2009); 9. o manifesto em defesa à avaliação psicológica (Noronha \& 
cols, 2002) e 10. o relatório sobre a avaliação dos testes psicológicos (CFP, 2004).

O investimento na área de avaliação psicológica e os seus impactos podem ser observados à medida que se examina a produção do I Congresso Brasileiro de Psicologia, em 2002. Noronha, Vendramini e Freitas (2004) levantaram entre os trabalhos apresentados na área de avaliação que 50\% estavam relacionados à construção, validação, padronização e adaptação de instrumentos, sendo que esses dados demonstraram a preocupação de tornar os instrumentos de medidas mais seguros e confiáveis. Mais recentemente, Joly, Silva, Nunes e Souza (2007) analisaram a produção científica em painéis de congressos em avaliação psicológica e os resultados apontaram para uma crescente publicação de estudos que buscaram aferir parâmetros psicométricos, quais sejam, evidências de validade, precisão e normatização aos instrumentos de medidas.

Outro impacto pode ser observado no IV Congresso Brasileiro de Avaliação Psicológica, V Congresso da Associação Brasileira de Rorschach e Métodos Projetivos e XIV Conferência Internacional de Avaliação Psicológica: Formas e Contextos ocorrido no ano de 2009 na cidade de Campinas. Esse evento permitiu a interface de trabalhos de pesquisadores e profissionais brasileiros, portugueses, espanhóis, americanos e africanos, o que evidencia a preocupação com o desenvolvimento da AP e dos testes psicológicos em âmbito nacional e internacional (IBAP, 2009).

A discussão sobre a avaliação psicológica recai sobre as ferramentas que podem ser utilizadas durante o processo, em especial, os testes psicológicos e na competência técnica do profissional responsável. Relação compreensível haja vista que quanto mais competência técnica o profissional tiver mais condições de selecionar o(s) teste(s) psicológico(s) adequado(s) para a sua finalidade. Entretanto, os testes psicológicos carecem de investimentos para suprir as lacunas, muito embora, a literatura nacional sinalize avanços.

O teste psicológico se constitui uma ferramenta de uso exclusivo do psicólogo e, segundo a Resolução CFP n. ${ }^{\circ}$ 02/2003, trata-se de um procedimento sistemático de observação e evidências de comportamentos e respostas das pessoas. Ele tem como objetivo a descrição e mensuração das especificidades individuais e processos psicológicos, abrangendo áreas como emoção/afeto, cognição/inteligência, motivação, personalidade, psicomotricidade, atenção, memória, percepção, dentre outras. 
Estudos apontam que os testes psicológicos, destinados à orientação profissional, à mensurar inteligência e/ou à personalidade, não apresentam informações sobre o autor, a data de publicação do manual e alguns nem referências, além da ausência de informações pertinentes aos estudos de padronização, validade e precisão (Noronha, Sartori, Freitas \& Ottati, 2001; Noronha, Freitas \& Ottati, 2002, 2003; Noronha \& Vendramini, 2003) o que pode vir a comprometer a seleção e o uso por parte dos profissionais. Os testes de personalidade apresentam menos informações sobre validade, precisão e normatização comparada aos testes de inteligência (Noronha, Freitas \& Ottati, 2003; Noronha e cols., 2003; Noronha \& Vendramini, 2003).

Nessa mesma linha, Vendramini e Lopes (2008) estudaram as dificuldades e diferenças de leitura associadas aos tópicos de Avaliação Psicológica e informações estatísticas contidas em manuais de testes. Os dados evidenciaram que mesmo que muitos profissionais revelem não possuir dificuldades na correção dos instrumentos psicológicos, estes constatam a falta de clareza nas informações de interpretação dos resultados. Tais evidências endossam a necessidade constante de avaliação dos instrumentos no que diz respeito às qualidades psicométricas e às exigências das regulamentações.

Ainda que, as qualidades psicométricas dos instrumentos de medida estejam em pleno desenvolvimento, a investigação feita por Noronha, Padilha e Fagan (2007) revelou que a maior parte dos pesquisados não faz uso de testes psicológicos em sua prática profissional. Esse dado pode justificar o não conhecimento de muitos instrumentos (Noronha \& Freitas, 2005), embora, o conhecimento de vários testes psicológicos não garanta o uso frequente dos testes bem como a competência do profissional. De todo modo, entende-se que o maior acesso aos testes possibilita contato com os conteúdos específicos da AP, ou seja, aplicação, avaliação e interpretação dos resultados (Noronha \& Freitas, 2005) e, consequentemente, com a psicometria e a estatística.

Atualmente, o acesso aos testes psicológicos que podem ser utilizados no processo de avaliação psicológica no Brasil pode ser consultado via site do Sistema de Avaliação de Testes Psicológicos - SATEPSI. Além do acesso a quais instrumentos existem no cenário brasileiro, há outras informações tais como os instrumentos que não são considerados testes psicológicos, os critérios de avaliação dos testes psicológicos, os membros da comissão e pareceristas, o relatório sobre os testes psicológicos, as resoluções, a avaliação dos testes 
psicológicos que são considerados favoráveis e desfavoráveis para uso, entre outras (SATEPSI, 2011).

Esse sistema favorece a seleção dos testes psicológicos, pois vai ao encontro da Resolução CFP n.0 02/2003, ao afirmar que o psicólogo deverá recorrer aos testes considerados favoráveis, salvo os casos de pesquisa. Ao contrário disso, será considerada falta ética, conforme disposto no Código de Ética Profissional do Psicólogo (2005).

A resolução de número do CFP 02/2003 está em consonância com 020 artigo do Código de Ética Profissional do Psicólogo (2005, p. 10), ao afirmar que 'é vedado ao psicólogo prestar serviços ou vincular o título de psicólogo a serviços de atendimento psicológico cujos procedimentos, técnicas e meios não estejam regulamentados ou reconhecidos pela profissão; emitir documentos sem fundamentação e qualidade técnico-científica; e interferir na validade e fidedignidade de instrumentos e técnicas psicológicas, adulterar seus resultados ou fazer declarações falsas'.

Essa resolução pressupõe que o psicólogo tenha uma postura ativa e crítica, pois afirma que 'o psicólogo que utiliza testes psicológicos como instrumento de trabalho, deve observar as informações contidas nos respectivos manuais e buscar informações adicionais para maior qualificação no aspecto técnico operacional do uso do instrumento, sobre a fundamentação técnica referente ao construto avaliado, sobre pesquisas recentes realizadas com o teste, além de conhecimentos de Psicometria e Estatística'. Portanto, o psicólogo, antes de tudo, é responsável pelo uso apropriado e pela interpretação correta dos escores de testes, sendo que essa responsabilidade é também observada em publicações internacionais (American Educational Research Association - AERA, American Psychology Association - APA \& National Council on Measurement in Education NCME, 1999; International Test Commission - ITC, 2003).

Diante da importância dos testes psicológicos no processo de avaliação psicológica, da existência do SATEPSI que compila diversas informações acerca da $A P$, esse artigo se propõe a levantar os motivos, segundo a resolução do Conselho Federal de Psicologia - CFP n.0 002/2003, pelos quais os testes psicológicos receberam Parecer desfavorável. 


\section{MÉTODO}

Fonte dos dados

Durante o primeiro semestre de 2010, as informações foram coletadas, por meio de 59 testes psicológicos presentes na lista dos testes que receberam Parecer desfavorável, disponibilizada no site http://www2.pol.org.br/satepsi/sistema/pareceres/desfavoraveis.htm. Destes, 07 testes foram apresentados por duas vezes à Comissão Consultiva e receberam novamente Parecer desfavorável, a saber: Inventário da Atitude de Trabalho - IAT; Suplemento para o Teste de Apercepção Temática - CAT-S; Teste de Apercepção Infantil com Figuras de Animais - CAT-A; Teste de Apercepção Infantil com Figuras Humanas - CAT-H; SAT; Teste de Personalidade - 16 PF; e Teste do Desenho Wartegg). Os outros testes foram: Teste de Personalidade - 16 PF; Teste de Personalidade - 16 PF revisado; Teste de Atenção Concentrada, Rapidez e Exatidão - ACRE; Bateria de Testes de aptidões Gerais II - BTAG II; Bateria fatorial CEPA (memória visual série A e B); Teste de Apercepção Infantil com Figuras Humanas - CAT - $\mathrm{H}$; Teste de Apercepção Infantil com Figuras Humanas - CAT- H revisado; Suplemento para o Teste de Apercepção Temática - CAT-S; Teste de Apercepção Infantil com Figuras de Animais - CAT - A; Teste de Apercepção Infantil com Figuras de Animais - CAT A Revisado; Cubos de Kohs; Teste Dominós 48 - D-48; Escala de Preconceito Profissional - EPP; Escala de Sociabilidade e Emotividade - ESE; Escala Reduzida de Autoconceito - ERA; Escore de Deterioração do Desenho da Pessoa - EDDP; Figuras Complexas de Rey; Inventário de Ansiedade Traço-Estado - IDATE-C; IDATE; Inventário da Atitude de Trabalho - IAT; Inventário de Autoanálise de Interesses Profissionais - IAIP; Inventário de Interesses de Kuder; Inventário de Interesses de L.L. Thurstone; Manual Prático de Avaliação do HTP e Família; Matrizes Progressivas de Raven - Escala Avançada; Método Gohmes; O Inventário Ilustrado de Interesses - GEIST; O Questionário Desiderativo; Palográfico; Prova de Nível Mental; Psicosônica - Teste Aleatorizado 017programador; Questionário Desiderativo (Ana Maria Cabrera); Reprodução de Figuras - RF; RF revisado; Teste de Raciocínio Lógico Numérico - RLN; Teste Raven de Operações Lógicas - RTLO; Teste de Apercepção Temática para Idosos e Adultos - SAT; SAT Revisado; Teste de Apercepção Temática-Suplemento CAT-S; Testes dos Conjuntos Emparelhados - TCE; Teste de Compreensão Técnico-Mecânico - TCTM; Teste Catálogo Livros Bressa Tramer; Teste D-70; 
Teste Dadahie - Dominância, agressividade, deferência, autonomia, humildade, intracepção-extracepção e encocatéxis-exocatéxis; Teste das Cores; Teste de Agradabilidade Básica - OPK; Teste de Aptidão a Mecânica/Aptidão Técnica TAM; Teste de Capacidade Intelectual; Teste de Frustração; Teste de Maturidade em Leitura; Teste Diagnóstico de Habilidades DHP; Teste de Organização Percepto-Motora - TOP; Teste de Prontidão para Leitura - TPL; Teste Projetivo Ômega - TPO; Teste de Sondagem Intelectual - TSI; Teste do Desenho Wartegg; Teste do Desenho - Wartegg Revisado e Zulliger.

\section{Material}

Uma planilha para organizar as informações contidas a partir dos critérios de análise dos testes psicológicos constantes nos Pareceres desfavoráveis. As informações colhidas foram o motivo do Parecer desfavorável, mês e ano de submissão, editora, responsável técnico, informações sobre número da edição, fundamentação teórica, existência de estudos de validade, precisão e normatização.

\section{Procedimento}

Os 59 pareceres foram impressos, consultados e analisados por dois juízes, independentemente, e obtiveram 95\% de concordância. Cabe mencionar que nos Pareceres foram também levantadas algumas informações cuja categoria de análise foi considerada como 'outros motivos', sendo que esta categoria se referia a aspectos que, embora não tenham sido centrais como possíveis motivos da reprovação, mereceriam um melhoramento.

\section{RESULTADOS E DISCUSSÃO}

Quanto aos motivos da reprovação, $04(6,8 \%)$ pareceres especificavam que o teste estava sem condição de uso e comercialização e $55(93,2 \%)$ estava sem condição de uso. Na tabela 1, é possível identificar que o ano de 2003 concentra o maior número de submissões dos testes psicológicos à comissão. Dado esse que pode ser justificado pela tentativa de as editoras e/ou responsáveis técnicos adequarem os testes às exigências da resolução 02/2003. 
Tabela 1.

Distribuição dos anos que os testes foram submetidos ao CFP segundo consta no parecer.

\begin{tabular}{ccc}
\hline Anos & $F$ & $\%$ \\
\hline 2001 & 3 & 5,1 \\
2002 & 3 & 5,1 \\
2003 & 29 & 49,2 \\
2004 & 17 & 28,8 \\
2006 & 7 & 11,9 \\
Total & 59 & 100 \\
\hline
\end{tabular}

Dos 59 testes analisados, 34 (57,6\%) eram da editora CEPA, 07 (11,9\%) da Casa do Psicólogo, 07 (11,9\%) da Editora Vetor, 08 (13,6\%) da editora Livro Pleno, $01(1,7 \%)$ do Centro Editor de Teste e Pesquisa em Psicologia - CETEPP, $01(1,7 \%)$ da Edites e $01(1,7 \%)$ não especificava a editora. Observou-se nos pareceres que $59,3 \%(n=35)$ dos testes descreviam no manual o responsável técnico.

Outro dado levantado nos pareceres foi se o teste informava a sua edição. Sendo assim, $47(79,7 \%)$ não informavam o número da edição, $02(3,4 \%)$ informavam que era a $1^{\text {a }}$ edição, $07(11,9 \%)$ a $2^{a}$ edição, $01(1,7 \%)$ a $3^{a}$ edição e $02(3,4 \%)$ a $5^{a}$ edição. Apesar de alguns testes terem sido editados mais de uma vez, nota-se que não há investimento devido para garantir o seu uso e consequente comercialização.

Um dos aspectos relevantes em um manual é a definição do construto, pois é um dos critérios relevantes para que o psicólogo selecione qual teste psicológico usar. De acordo com a Tabela 2 nota-se que aproximadamente metade dos testes com parecer desfavorável não apresentava referências mínimas para a fundamentação do construto a ser medido.

Tabela 2.

Dados relativos à fundamentação teórica dos testes com parecer desfavorável.

\begin{tabular}{ccc}
\hline Categorias acerca da fundamentação & $F$ & $\%$ \\
\hline Apresentavam fundamentação e definição de construto & 32 & 54,2 \\
Insuficiência de fundamentação & 14 & 23,7 \\
Ausência de definição de construto & 10 & 16,9 \\
Inconsistência de fundamentação & 03 & 5,1 \\
\hline Total & 59 & 100 \\
\hline
\end{tabular}


Dentre todos os atributos psicométricos de um instrumento, a validade é o mais importante, pois ela evidencia se o instrumento mensura o que se propõe. Em outras palavras, se não houver evidências dessa natureza não há segurança de que as interpretações sobre as características psicológicas das pessoas sugeridas pelas respostas sejam legítimas (Primi, Nascimento Souza \& 2004). Conforme evidencia a Tabela 3, a maioria dos testes que recebeu parecer desfavorável apresenta estudos sobre a sua validade, mas com informações insuficientes que legitime o seu uso.

Tabela 3.

Categorias relativas à validade dos testes com parecer desfavorável.

\begin{tabular}{ccc}
\hline Categorias levantadas em validade & $F$ & $\%$ \\
\hline Ausência de estudos brasileiros & 20 & 33,9 \\
Ausência de estudos & 14 & 23,7 \\
Ausência de estudos atualizados & 08 & 13,6 \\
Outros & 04 & 6,8 \\
Apresentam estudos & 03 & 5,1 \\
Estudo desatualizado & 03 & 5,1 \\
Ausência de parâmetros psicométricos e estudos & 03 & 5,1 \\
atualizados & 02 & 3,4 \\
Ausência de estudos psicométricos & 02 & 3,4 \\
\hline Incompleto & 59 & 100 \\
\hline
\end{tabular}

Em um teste psicológico é também importante evidências de que a medida seja confiável ou precisa. Caso não haja informações a respeito, não é possível mensurar quanto os resultados mudariam se fossem dadas novas oportunidades de avaliação, tais como outro profissional avaliando o mesmo sujeito, empregando o mesmo método, ou outro instrumento e/ou oportunidade com os mesmos objetivos (Primi, Nascimento \& Souza, 2004). Conforme evidencia a Tabela 4, a maioria dos testes que recebeu parecer desfavorável apresenta estudos sobre a sua precisão, embora com informações insuficientes que assegurem os resultados obtidos. 
Tabela 4.

Categorias relativas à precisão dos testes com parecer desfavorável.

\begin{tabular}{rcc}
\hline Categorias levantadas em precisão & $F$ & $\%$ \\
\hline Ausência de estudos brasileiros & 16 & 27,1 \\
Ausência de estudos & 14 & 23,7 \\
Apresentam estudos & 8 & 13,6 \\
Ausência de estudos atualizados & 8 & 13,6 \\
Estudo desatualizado & 3 & 5,1 \\
Ausência de parâmetros psicométricos e estudos atualizados & 3 & 5,1 \\
Incompleto & 3 & 5,1 \\
Outros & 3 & 5,1 \\
Ausência de estudos psicométricos & 1 & 1,7 \\
\hline Total & 59 & 100 \\
\hline
\end{tabular}

Todo instrumento deve ter estudo de normatização no qual se estabelece critérios padronizados que englobam a aplicação, correção e interpretação dos dados como também deve oferecer tabelas normativas que permitam a comparação dos resultados de diferentes pessoas. Caso não haja, o profissional terá dificuldades nas interpretações dos resultados, já que as pessoas serão comparadas com expectativas inapropriadas a nossa realidade (Primi, Nascimento \& Souza, 2004). Na Tabela 5, é possível constatar que, a maioria dos testes que recebeu parecer desfavorável apresenta estudos sobre a normatização o que auxilia o profissional a aplicar, corrigir e interpretar os dados obtidos do respondente.

Tabela 5.

Categorias relativas à normatização dos testes com parecer desfavorável.

\begin{tabular}{ccc} 
Categorias levantadas em normatização & $F$ & $\%$ \\
\hline Apresentam estudos & 40 & 67,8 \\
Ausência de estudos brasileiros & 6 & 10,2 \\
Estudo desatualizado & 5 & 8,5 \\
Ausência de estudos & 3 & 5,1 \\
Incompleto & 3 & 5,1 \\
Amostra desatualizada & 2 & 3,4 \\
\hline Total & 59 & 100,0
\end{tabular}

Por fim, a leitura dos pareceres permitiu levantar uma categoria de análise denominada 'outros motivos'. Cabe lembrar que essa categoria abarcou aspectos que sinalizassem a necessidade do teste psicológico se adequar às normas da 
resolução 02/2003 (CFP). Portanto, nota-se na tabela 6 que, a grande maioria dos testes psicológicos não preencheu esse requisito, embora haja testes que não apresentam referências os quais tenha se pautado ou se apresenta, menciona referências antigas, evidenciando assim o descompromisso com o desenvolvimento científico. Dados esses que corroboram com os resultados obtidos por Noronha e cols. (2001) nos testes de inteligência.

Tabela 6.

'Outros motivos' para que os testes psicológicos, com parecer desfavorável, se adequem a resolução 02/2003.

\begin{tabular}{ccc}
\hline Outros motivos que careceriam de melhoramentos & $F$ & $\%$ \\
Não houve outros motivos & 31 & 52,6 \\
Ausência de análise de itens & 7 & 11,8 \\
Ausência de referências bibliográficas e/ou há somente & 7 & 11,8 \\
referências antigas & 7 & 11,8 \\
Não ha informações relevantes & 4 & 6,8 \\
Estudos desatualizados & 3 & 5,1 \\
Outros (falhas metodológicas, não possui manual, faltam \\
dados da adaptação) & 59 & 100 \\
\hline Total & 5
\end{tabular}

De maneira geral, 59 testes psicológicos, que receberam parecer desfavorável estão sem condições de uso e consequentemente comercialização. Esses motivos são justificados, especialmente, pelo fato desses testes não privilegiarem dois dos três indicadores que compõem as propriedades mínimas de um teste psicológico, a saber, a validade e precisão. Esse mesmo dado pode ser encontrado nos estudos de Noronha, Sartori, Freitas e Ottati, (2001); Noronha, Freitas \& Ottati (2003) e Primi, Nascimento e Souza (2004). Nota-se que o terceiro indicador, a normatização recebeu maior investimento, pois está presente na maioria dos testes com parecer desfavorável sugerindo que os pesquisadores/editoras priorizam aspectos como a aplicação, correção e interpretação dos testes em detrimento das evidências de validade e precisão.

Desse modo, o parecer desfavorável impede que o teste psicológico possa ser usado por psicólogos em sua prestação de serviços, mas não impede, em absoluto, o uso do teste como objeto de estudo em uma pesquisa (Primi, Nascimento Souza, 2004). Dito de outra maneira, as informações acerca dos pareceres desfavoráveis contribuem para a exclusão do uso do teste em qualquer contexto profissional, mas pode direcionar futuras pesquisas. 


\section{CONSIDERAÇÕES FINAIS}

Nos últimos anos, os psicólogos brasileiros têm focado a área de AP, como evidenciam as publicações. Esse investimento se justifica, uma vez que a AP permeia as áreas de atuação da Psicologia desde as mais tradicionais como a clínica, escolar, organizacional como outras que estão em plena expansão, tais como a jurídica, a neuropsicologia, a hospitalar, trânsito, entre outras.

Sob essa perspectiva, tentar compreender os motivos que levaram alguns testes psicológicos a não preencherem os requisitos mínimos para serem usados e comercializados, nos leva a refletir sobre nosso instrumental de trabalho. Por um lado, esse panorama (dos testes não atenderem aos requisitos mínimos) em meados de 2000 era aceitável, mas atualmente, deparar-se com essa informação é lamentável, pois parece emergir o descaso de responsáveis no nãoinvestimento em ferramentas que são úteis a prestação de serviços - os testes psicológicos. Por outro lado, pode estar havendo o movimento de investir em novos testes psicológicos ao invés de reavaliar os antigos. Esse dado pode ser endossado diante dos novos testes que são inseridos, periodicamente, na lista dos testes com parecer favorável conforme atualizações no site do SATEPSI.

Mesmo que os avanços sejam notórios, seria ingênuo não mencionar que o uso dos testes psicológicos ainda enfrenta resistência por parte de alguns psicólogos (Noronha \& cols, 2002). Esse fato pode ser entendido quando se considera que há críticas contundentes em relação às ementas e às propostas de ensino dos testes psicológicos em muitas universidades brasileiras. Entende-se que a formação inadequada na área depõe de forma negativa na vida profissional do futuro psicólogo. Diante desse quadro, por agora, é mais confortante saber que há uma comissão julgadora que avalia os testes psicológicos para que o profissional interessado possa utilizá-lo com maior segurança em sua prática profissional.

Todavia, entende-se que esse movimento não é suficiente. É necessário que os futuros psicólogos e aqueles que querem lançar mão de testes psicológicos em sua atuação profissional tenham uma compreensão do que ele pretende avaliar, como foi construído, para qual público está direcionado, como aplicar e corrigir para atuar de forma ética e responsável. Investir na formação do futuro profissional e/ou propor cursos de especialização parece atingir o problema no alvo, conforme iniciativas do IBAP, por exemplo, ao sugerir o curso de especialização em avaliação psicológica. 
Ao investir na formação, garante as ferramentas teóricas e técnicas ao profissional permitindo que ele se torne menos dependente, ou seja, menos regulado por conselho(s) regionais e/ou federal. Iniciativas essas que demonstram uma 'nova era' da Avaliação psicológica que iniciou em meados de 2000 e culmina nesse ano de 2011, com o ano temático sobre a avaliação psicológica com direito a um blog: anotematico.cfp.org.br/2011/.

\section{REFERÊNCIAS}

American Educational Research Association, American Psychological Association \& National Council on Measurement in Education (1999). Standards for Educational and Psychological Testing. New York: American Educational Research Association.

Código de Ética profissional do Psicólogo (2005). Código de ética profissional do Psicólogo. Conselho Federal de Psicologia - CFP. Retirado em 10 de fevereiro de 2007 do site http://www.pol.org.br/pol/export/sites/default/pol/legislacao/legislacaoDoc umentos/codigo_etica.pdf.

Instituto Brasileiro de Avaliação Psicológica (2009). Congresso 2009. [citado em 26 de janeiro de 2009]. Disponível em: www.ibapnet.org.br/congresso2009/index.html

Joly, M. C. R. A., Silva, M. C. R. da, Nunes, M. F. O. \& Souza, M. S. (2007). Análise da produção científica em painéis dos congressos brasileiros de avaliação psicológica. Avaliação Psicológica, 6(2), 239-252.

International Testing Commission (2003). Diretrizes para o uso de Testes: International Testing Commission. Brasil: Instituto Brasileiro de Avaliação Psicológica e Conselho Federal de Psicologia. Tradução de Solange Wechsler, Nilton Barbosa, Patrícia Schelini, Mônica Gobitta, Patrícia Pereira e Nilza Catini.

Noronha, A. P.P. \& Freitas, F. A. (2005). Testes psicológicos,usos e conhecimento. Psico, 36(1), 21-28.

Noronha, A. P. P., Vendramini, C. M. M. (2003). Parâmetros psicométricos: estudo comparativo entre testes de inteligência e de personalidade. Psicologia: Reflexão e Crítica, 16(1), 177-182.

Noronha, A. P. P., Freitas, F. A. \& Ottati, F. (2002). Parâmetros psicométricos de testes psicológicos de inteligência. Interação em Psicologia, 6(2), 195-201. 
Noronha, A. P. P., Freitas, F. A. \& Ottati, F. (2003). Análise de instrumentos de avaliação de interesses profissionais. Psicologia: Teoria e Pesquisa, 19(3), 287-291.

Noronha, A. P. P., Sartori, F. A.; Freitas, F. A. \& Ottati, F. (2003). Informações contidas nos manuais de Inteligência publicados no Brasil. Psicologia em Estudo, 6(2), 101-106.

Noronha, A. P. P., Padilha, S. \& Fagan, C. Z. (2007). Instrumentos de Avaliação Psicológica: uso e parecer de psicólogos. Avaliação Psicológica, 6(1), 69-76.

Noronha, A. P. P., Vendramini, C. M. M., \& Freitas, F. A. (2004). Avaliação Psicológica no I Congresso Brasileiro de Psicologia. Em C. Machado, L. S. Almeida, M. Gonçalves \& V. Ramalho (Orgs.), Avaliação Psicológica: Formas e Contextos (pp.11-15). Braga: Psiquilíbrios Edições.

Oakland, T. (2004). Use of educational and psychological tests internationally. Applied Psychology: An International Review, 53(2), 157-172.

Pasquali, L. (2003). Psicometria: teoria dos testes na psicologia e na educação. Petrópolis: Vozes.

Pasquali, L., \& Alchieri, J. C. (2001). Os testes psicológicos no Brasil. Em L. Pasquali (Org.), Técnicas de Exame Psicológico - TEP: Manual, volume 1 Fundamentos das Técnicas Psicológicas (pp. 197-220). São Paulo: Casa do Psicólogo.

Primi, R., Nascimento, R. S. G. F.,\& Souza, A. S.(2004). Critériospara avaliação de testes psicológicos. Em Conselho Federal de Psicologia - CFP (Prg.). Avaliação dos testes psicológicos: relatório (pp. 31-55). Brasília: CFP.

Sistema de Avaliação de Testes Psicológicos - SATEPSI (2011). Sistema de Avaliação dos Testes Psicológicos. Retirado em 10 de janeiro de 2010, do site http://www2.pol.org.br/satepsi/sistema/admin.cfm

Urbina, S. (2007). Fundamentos da testagem psicológica. Porto Alegre: Artmed.

Vendranini, C. M. M. \& Lopes, F. L. (2008). Leitura de manuais de testes psicológicos por estudantes e profissionais de psicologia. Avaliação Psicológica, 7(1), 93-105.

Contato: ferfreitas@ig.com.br

Recebido em: 27/04/2011

Revisado em: 16/05/2011

Aceito em: 08/06/2011 